\title{
Soy Phytoestrogens on DNA Methylation in Prostate Cancer
}

Received: March 21, 2017; Accepted: March 27, 2017; Published: March 31, 2017

Soy phytoestrogens are dietary components with considerable effects on reducing the incidence of prostate cancer. Furthermore, the epigenetic regulation of gene expression can be modified by soy phytoestrogens [1]. Qualitative and quantitative studies show a decrease of promoter methylation on tumour suppressor gene such as BRCA1, BRCA2, EPHB2, GSTP1 and RASSF1A on prostate cancer cell lines treated with genistein and daidzein. Effects of both molecules were compared to the demethylating agent of DNA, the 5 -azacytidin [2,3]. Indeed, these genes are known to be hypermethylated in prostate cancer. This hypermethylation leads to the loss of their expressions and to chemotherapy-resistance of tumor cells $[4,5]$.

In order to understand the molecular mechanisms involved in the methylation reversion of DNA by phytoestrogens, a comparative study of the effect of phytoestrogens and $17 \beta$-estradiol was carried out on prostate cancer cell lines PC-3, LNCaP and DU 145 which differ in their Androgen Receptor (AR) status but share Estrogen Receptor Beta (ER $\beta)$. For this purpose, 24 genes were selected for their direct involvement in prostate cancer. This gene panel compared the effects of genistein, daidzein, 17 $\beta$-estradiol and 5-azacytidine. A demethylating effect of genistein and daidzein on most genes was observed except for the DLC1 and hsa-miR-34a genes in the PC-3; DLC1, hsa-miR-34a and MSX1 in DU 145 and OPCML in LNCaP [6].

Furthermore, $17 \beta$-estradiol effect on gene demethylation was similar to 5-azacytidine and soy phytoestrogens, it reduces the methylation of oncosuppressors in prostate cancer. This result suggests that demethylating action of phytoestrogens, which have a similar molecular structure to estrogens, would pass through ER $\beta$. However, it is possible that some of phytoestrogen effects on DNA methylation operate by other pathways because all genes don't have Estrogen Response Elements (EREs) in their regulatory regions. Indeed, phytoestrogens-ER $\beta$ complex is translocated in nucleus where it binds to the EREs on target genes promoters in order to moduce their demethylating effect in cells. In addition, methylome study by MeDIP-on-chip technique on DU 145 and LNCaP cells after treatment with genistein, daidzein and 5 -azacytidine showed 88 genes modulated by soy phytoestrogens in DU 145 against 478 genes in LNCaP. MAD1L1, TRAF7, KDM4B and $h T E R T$ genes were commonly differentially methylated in both cell lines after treatment and demethylating effect of genistein and daidzein was more marked than 5-azacytidine [7]. These demethylating effects imply a transcription regulation of these genes by DNA methylation. Signaling pathways analysis of these 4 genes reveals an association with the NF-kB and $p 53$ pathways.

\author{
Marine Daures ${ }^{1,2}$, Marjolaine \\ Ngollo ${ }^{1,2}$, \\ Mouhamed Idrissou ${ }^{1,2}$, \\ Gaëlle Judes ${ }^{1,2}$, \\ Khaldoun Rifaï ${ }^{1,2}$, \\ Frédérique Penault-Llorca ${ }^{2,3}$, \\ Yves Jean Bignon ${ }^{1,2}$, \\ Laurent Guy ${ }^{2,4}$ and \\ Dominique Bernard- \\ Gallon $^{1,2^{*}}$
}

1 Department of Oncogenetics, Centre Jean Perrin, 63001 Clermont-Ferrand, France

2 University Clermont Auvergne, INSERM U1240, IMoST, 63000 Clermont-Ferrand, France

3 Department of Biopathology, Centre Jean Perrin, 63000 Clermont-Ferrand, France

4 Department of Urology, CHU Gabriel Montpied, 63000 Clermont-Ferrand, France

Corresponding author:

Dominique Bernard-Gallon

” dominique.gallon-bernard@clermont. unicancer.fr

Department of Oncogenetics, Centre Jean Perrin, INSERM U1240, Clermont-Ferrand, France.

Tel: 0473178358

Citation: Daures $\mathrm{M}$, Ngollo $\mathrm{M}$, Idrissou $M$, et al. Soy Phytoestrogens on DNA Methylation in Prostate Cancer. J Clin Epigenet. 2017, 3:1.

These two pathways are deregulated in prostate cancers and play a major role in proliferation and apoptosis. Previous studies have shown a decrease in NF-kB activity by genistein in prostate cancer cell lines suggesting an indirect effect of genistein on tumor proliferation [8]. 
Mitotic arrest deficient-like 1 (MAD1L1) is involved in the cell cycle by controlling the mitotic spindle-assembly and chromosome alignment. The expression loss of this gene contributes to tumor development. This result suggests a reactivation of MAD1L1 gene expression by promoter demethylation by phytoestrogens.

Interplay between TNF Receptor-associated factor 7 (TRAF7) and p53 was demonstrated in breast cancer and it shows a decrease in TRAF7 expression [9]. TRAF7 impair p53 via its E3 ubiquinine ligase activity. Decrease of TRAF7 expression leads to an accumulation of $\mathrm{p} 53$ protein in tumor cells causing a proliferation increase. Decrease of TRAF7 methylation with genistein and daidzein would increase transcription of this gene in prostate cancer cells, reflecting the anti-proliferative role of soy phytoestrogens in cancer.

Lysine (K)-specific demethylase $4 B(K D M 4 B)$ and telomerase reverse transcriptase ( $h T E R T)$ possess oncogenic activity in cancers [10-12]. KDM4B overexpression in prostate cancer and its association with the Androgen Receptor (AR) suggest that KDM4B is a potential factor in tumor progression [13]. KDM4B acts as a coactivator of $A R$, increasing its transcriptional activity and stability by inhibiting its proteasome degradation [14]. Thus, KDM4B promoter does not show methylation, which leads to suppose that gene regulation is not dependent on DNA methylation. These observations may explain the work of Coffey et al. which shows a KDM4B increase in prostate cancers. However, genistein and daidzein treatments show an increase on KDM4B and hTERT methylation. These results suggest a methylation effect of soy phytoestrogens on genes with an oncogenic effect. Genistein effect on repression of $h T E R T$ transcription has been previously demonstrated. Indeed, genistein would indirectly inhibit hTERT repression by negatively regulating $C-M Y C$ transcription factor which activate $h T E R T$ transcription [15].

These results confirm the major role of epigenetic mechanisms in tumor development and suggest that soy phytoestrogens behave like epidrugs in prostate cancers. 


\section{References}

1 Karsli-Ceppioglu S, Ngollo M, Judes G, Penault LF, Bignon YJ, et al. (2015) The Role of Soy Phytoestrogens on Genetic and Epigenetic Mechanisms of Prostate Cancer. Enzymes 37: 193-221.

2 Adjakly M, Bosviel R, Rabiau N, Boiteux JP, Bignon YJ, et al. (2011) DNA methylation and soy phytoestrogens: quantitative study in DU145 and PC-3 human prostate cancer cell lines. Epigenomics 3: 795803.

3 Vardi A, Bosviel R, Rabiau N, Adjakly M, Satih S, et al. (2010) Soy phytoestrogens modify DNA methylation of GSTP1, RASSF1A, EPH2 and BRCA1 promoter in prostate cancer cells. In Vivo 24: 393-400.

4 Majumdar S, Buckles E, Estrada J, Koochekpour S (2011) Aberrant DNA methylation and prostate cancer. Curr Genomics 12: 486-505.

5 Ngollo M, Dagdemir A, Karsli-Ceppioglu S, Judes G, Pajon A, et al. (2014) Epigenetic modifications in prostate cancer. Epigenomics 6 : 415-426.

6 Adjakly M, Ngollo M, Lebert A, Dagdemir A, Penault-Llorca F, et al. (2014) Comparative effects of soy phytoestrogens and 17betaestradiol on DNA methylation of a panel of 24 genes in prostate cancer cell lines. Nutr Cancer 66: 474-482.

7 Karsli-Ceppioglu S, Ngollo M, Adjakly M, Dagdemir A, Judes G, et al. (2015) Genome-wide DNA methylation modified by soy phytoestrogens: role for epigenetic therapeutics in prostate cancer? Omics 19: 209-219.
8 Pavese JM, Farmer RL, Bergan RC (2010) Inhibition of cancer cell invasion and metastasis by genistein. Cancer Metastasis Rev 29: 465482.

9 Wang L, Zhang S, Qu G, Zhang D, Li S, et al. (2013) Downregulation of ubiquitin E3 ligase TNF receptor-associated factor 7 leads to stabilization of p53 in breast cancer. Oncol Rep 29: 283-287.

10 Toyokawa G, Cho HS, Iwai Y, Yoshimatsu M, Takawa M, et al. (2011) The histone demethylase JMJD2B plays an essential role in human carcinogenesis through positive regulation of cyclin-dependent kinase 6. Cancer Prev Res (Phila) 4: 2051-2061.

11 Artandi SE, Alson S, Tietze MK, Sharpless NE, Ye S, et al. (2002) Constitutive telomerase expression promotes mammary carcinomas in aging mice. Proc Natl Acad Sci U S A 99: 8191-8196.

12 Hahn WC, Stewart SA, Brooks MW, York SG, Eaton E, et al. (1999) Inhibition of telomerase limits the growth of human cancer cells. Nat Med 5: 1164-1170.

13 Chu CH, Wang LY, Hsu KC, Chen CC, Cheng HH, et al. (2014) KDM4B as a target for prostate cancer: structural analysis and selective inhibition by a novel inhibitor. J Med Chem 57: 5975-5985.

14 Coffey K, Rogerson L, Ryan-Munden C, Alkharaif D, Stockley J, et al. (2013) The lysine demethylase, KDM4B, is a key molecule in androgen receptor signalling and turnover. Nucleic Acids Res 41: 4433-4446.

15 Ouchi H, Ishiguro H, Ikeda N, Hori M, Kubota Y, et al. (2005) Genistein induces cell growth inhibition in prostate cancer through the suppression of telomerase activity. Int J Urol 12: 73-80. 